\title{
Caesarean Section Without Indwelling Catheter
}

Tahera Begum

M Jalal Uddin ${ }^{2 *}$

Ishrat Jabin

'Department of Gynaecology \& Obstetrics Chattagram Maa-O-Shishu Hospital Medical College Chittagong, Bangladesh.

${ }^{2}$ Department of Community Medicine \& Public Health Chattagram Maa-O-Shishu Hospital Medical College Chittagong, Bangladesh

\section{*Correspondence to:}

\section{Dr. M Jalal Uddin}

Professor of Community Medicine \& Public Health Chattagram Maa-O-Shishu Hospital Medical College Chittagong, Bangladesh.

Mobile : +8801819909464

E-mail:drialal65@gmail.com

\begin{abstract}
Background: Many studies were conducted worldwide on the subject but there is none in Chittagong. To get our experience we had conducted the study. Methods: It was an experimental study. 70 cases were conveniently selected under certain inclusion and exclusion criteria. Cases were managed by the obstetricians unrelated to the study. All cases were meticulously observed by the investigators and findings were instantly recorded. All data were manually managed. A discussion was made and conclusion was drawn. Results: Total 70 cases were studied. 35 primae and 35 multipara. Mean age was 27 years +2.3 . All were literate and $86 \%$ were housewives. Cases were without medical, surgical complications and were uneventfully managed. First voiding time was 4.28 hours +0.45 . $07 \%$ cases were evacuated with plain catheter after 7 hours for pain and bladder distention. Average hospital stay 2.3 days. There was no occurrence of urinary tract infection. Conclusion: Indwelling catheter should not be used unless it is strongly indicated.
\end{abstract}

Key words: LSCS; Indwelling catheter; Complications.

\section{INTRODUCTION}

Use of indwelling catheter in caesarean section is a routine practice. This is for better per operative exposure and to prevent post operative urinary retention but this indwelling catheter causes discomfort and urinary tract infection. It also prolongs hospital stay which costs more and there is more chance of nosocomial infection ${ }^{1}$. Avoiding indwelling catheter all problems could be avoided but this needs pre operative bladder evacuation, careful per-operative check up and post operative follow up. Urine analysis, culture sensitivity were done for all cases to detect urinary tract infection.

\section{MATERIALS AND METHODS}

01. Study title

02. Study type

03. Sampling technique

04. Sampling size

05. Inclusion, exclusion criteria

06. Study area

07. Study period

08. Ethical implication

09. Data management.

10. Statistical analysis
Caesarean section without indwelling catheter

Experimental study

Convenient sampling

70 (considering resource constraints

LSCS indicated but free from complications Chattagram Maa-O-Shishu Hospital Medical College January-March, 2014

Informed written consent from patient was taken

Manual management

$\mathrm{Z}$ test was used 


\section{RESULTS}

Total 70 cases were studied. 35 primae and 35 multipara. Mean age was 27 years $+/-2.3$. All were literate and $86 \%$ were housewives. Cases were without medical, surgical complications and were uneventfully managed. First voiding time was 4.28 hours +0.45 . Four cases $(5.7 \%)$ were evacuated with plain catheter only for one time after 7 hours of delivery for pain and bladder distention. Average hospital stays 2.3 days. There was no occurrence of injury and urinary tract infection.

Table 1: Post operative urinary bladder evacuation

\begin{tabular}{lc} 
Bladder evacuation & No. of $\operatorname{cases}(\%)$ \\
Without catheter & $66(94.3 \%)$ \\
With plain catheter & $04(05.7 \%)$ \\
Total & $70(100 \%)$ \\
\hline
\end{tabular}

\section{DISCUSSION}

Indwelling catheter is harmful. It causes urinary tract infection at different magnitude and health hazards depending on duration of catheterization. Moreover, it causes enormous discomfort and may cause injury to urethra. Aforesaid problems are preventable by avoiding indwelling catheter. Present study shows $5.7 \%$ cases needed plain catheterization only for one time 7 hours after caesarean section. Study conducted by them in 2001 also showed 5\% cases had needed catheterization ${ }^{2}$. Both the studies showed no occurrence of urinary tract infection. Another study showed $4.4 \%$ cases were evacuated with plain catheter during post operative period ${ }^{3}$. Urinary tract infection didn't occur in this study. First voiding time and average time of hospital are also similar. The findings are consistent with one another $(\mathrm{P}>.05)$. Besides health hazards catheterization also increases hospital bill.

\section{CONCLUSION}

Catheterization causes discomfort and health hazards. It increases hospital bill volume even without complications. So, catheterization should be avoided unless there is clean cut indication.

\section{DISCLOSURE}

All the authors declared no competing interest.

\section{REFERENCES}

1. Acharya et al. Caesarean section without urethral catheterization: A randomized controlled trial. Kathmandu university medical journal. 2012; 10(38) : 18-22.

2. Joseph et al. Use of indwelling urinary cather at caesarean delivery. Journal of Obstetrics and Gynaecology. 2001; 97(4):66.

3. Ghoreishi J. Indwelling urinary catheter in caesarean delivery. International journal on Gynae- Obstetrics. 2003; 83(3): 267-270. 